\title{
Familial aggregation analysis of gene expressions
} Shao-Qi Rao*†1,2,3, Liang-De Xu+1, Guang-Mei Zhang ${ }^{4}, \mathrm{Xia} \mathrm{Li}^{* 1,3,5}$, Lin Li², Gong-Qing Shen², Yang Jiang1, Yue-Ying Yang1, Bin-Sheng Gong1, Wei Jiang1, Fan Zhang1, Yun Xiao ${ }^{1}$ and Qing K Wang ${ }^{2}$

\author{
Address: ${ }^{1}$ Department of Bioinformatics, Harbin Medical University, Harbin 150086, People's Republic of China, ${ }^{2}$ Departments of Molecular \\ Cardiology and Cardiovascular Medicine, The Cleveland Clinic Foundation, 9500 Euclid Avenue, Cleveland, Ohio 44195, USA, 3 Biomedical \\ Engineering Institute, Capital University of Medical Sciences, Beijing 100054, People's Republic of China, ${ }^{4}$ The First Clinical College, Harbin \\ Medical University, Harbin 150081, People's Republic of China and ${ }^{5}$ Department of Computer Science, Harbin Institute of Technology, Harbin \\ 150080, People's Republic of China \\ Email: Shao-Qi Rao* - raos@ccf.org; Liang-DeXu - xuliangde@126.com; Guang-Mei Zhang - zhanggm@ems.hrbmu.edu.cn; \\ Xia Li* - lixia@ems.hrbmu.edu.cn; Lin Li - lil@ccf.org; Gong-Qing Shen - sheng@ccf.org; Yang Jiang - jybackup@163.com; Yue- \\ Ying Yang - yyy109_109109@sohu.com; Bin-Sheng Gong - gongbinsheng@gmail.com; Wei Jiang - bioccjw@126.com; \\ Fan Zhang - zhangfan@gmail.com; Yun Xiao - wintarcxy@yahoo.com.cn; Qing K Wang - wangq2@ccf.org \\ * Corresponding authors †Equal contributors
}

from Genetic Analysis Workshop 15

St. Pete Beach, Florida, USA. II-15 November 2006

Published: 18 December 2007

BMC Proceedings 2007, I (Suppl I):S49

This article is available from: http://www.biomedcentral.com/I753-656I/I/SI/S49

(c) 2007 Rao et al; licensee BioMed Central Ltd.

This is an open access article distributed under the terms of the Creative Commons Attribution License (http://creativecommons.org/licenses/by/2.0), which permits unrestricted use, distribution, and reproduction in any medium, provided the original work is properly cited.

\begin{abstract}
Traditional studies of familial aggregation are aimed at defining the genetic (and non-genetic) causes of a disease from physiological or clinical traits. However, there has been little attempt to use genome-wide gene expressions, the direct phenotypic measures of genes, as the traits to investigate several extended issues regarding the distributions of familially aggregated genes on chromosomes or in functions. In this study we conducted a genome-wide familial aggregation analysis by using the in vitro cell gene expressions of 3300 human autosome genes (Problem I data provided to Genetic Analysis Workshop 15) in order to answer three basic genetics questions. First, we investigated how gene expressions aggregate among different types (degrees) of relative pairs. Second, we conducted a bioinformatics analysis of highly familially aggregated genes to see how they are distributed on chromosomes. Third, we performed a gene ontology enrichment test of familially aggregated genes to find evidence to support their functional consensus. The results indicated that I) gene expressions did aggregate in families, especially between sibs. Of 3300 human genes analyzed, there were a total of I 105 genes with one or more significant (empirical $p<0.05$ ) familial correlation; 2 ) there were several genomic hot spots where highly familially aggregated genes (e.g., the chromosome 6 HLA genes cluster) were clustered; 3) as we expected, gene ontology enrichment tests revealed that the 1105 genes were aggregating not only in families but also in functional categories.
\end{abstract}




\section{Background}

Familial aggregation is the more frequent occurrence of a trait in members of a family than among non-related individuals. Thus, it is a common analysis method to determine the genetic contribution to a complex human disease. Technically, this type of analysis is a more detailed version of the mixed linear model approach in that each type of relative pairs is estimated separately instead of modeling them as a function of a few parameters in a single covariance matrix. Historically, familial aggregation analysis has been the most popular method for determining genetic causes of disease. This method, in essence, is to estimate the correlations between various biological relatives and then similarly assume that they can be parsimoniously explained by an additive genetic contribution and a common household contribution, but without making all the other assumptions of the mixed linear model. Although familial aggregation has been well studied for many diseases [1], genome-wide gene expressions typically have not been used as the traits. Problem 1 data for Genetic Analysis Workshop 15 (GAW15), initially used for mapping expression quantitative trait loci [2], provided expression levels of 3554 genes in lymphoblastoid cells for 14 three-generation CEPH (Centre d'Etude du Polymorphisme Humain) Utah families. Because of their inborn nature, expression of these genes might be less affected by a list of environmental factors for complex human diseases. Therefore, the specific aims of the present study were to answer three genetics questions: 1 ) how gene expressions aggregate among different types (degrees) of relative pairs; 2) how they are distributed on chromosomes; and 3) what functional implications they have.

\section{Methods}

\section{Description of the data set}

Expression levels of genes in lymphoblastoid cells of each individual of 14 three-generation CEPH Utah families ( $~ 8$ offspring per sibship, $\sim 14$ individuals per family, total of 194 individuals) were provided for GAW15 Problem 1. For 3554 of the 8500 genes tested, Morley et al. [2] found greater variation among individuals than between replicate determinations on the same individual. We further reduced the above number of genes to 3300 by deleting the genes having uncertain chromosome locations or situated on chromosomes $\mathrm{X}$ and $\mathrm{Y}$.

\section{Calculating familial correlations}

S.A.G.E FCOR [3] can be used to calculate familial correlations for a variety of biological relative types. Here, this module was used to calculate familial correlation $(R)$ for 15 relative types: father-son (FS), mother-son (MS), father-daughter (FD), mother-daughter (MD), brotherbrother (BB), sister-brother (SB), sister-sister (SS), grandfather-father-grandson (FFS), grandmother-father-grand- son (MFS), grandfather-mother-grandson (FMS), grandmother-mother-grandson (MMS), grandfatherfather-granddaughter (FFD), grandmother-father-granddaughter (MFD), grandfather-mother-granddaughter (FMD) and grandmother-mother-granddaughter (MMD). As reported by S.A.G.E. PEDINFO [3], the CEPH Utah families provided 220 parent-offspring pairs, 378 sibling pairs, and 440 extended relative pairs. To test the statistical significance of a correlation estimate and to correct for multiple tests for 15 relative types, we also performed 100,000 permutations on the $3300 \times 15$ (genes $\times$ number of relative types) matrix. The empirical thresholds are $R=$ 0.4609 and $R=0.6532$, respectively, for the significant levels of 0.05 and 0.01 .

\section{Gene ontology (GO) enrichment analysis of familially aggregated genes}

To see if the genes significantly aggregating in families are also aggregating in functional categories, we performed a gene ontology (GO) enrichment test. Suppose that a total of $N(3300)$ genes (set A) for the analyzed data are annotated in GO in which a set of M (1105 found in this study) genes (set B) are significantly familially aggregated. For a given GO category, a gene is either in the category or not in the category. Suppose further that $n$ genes out of set A and $m$ genes out of set B are in the category. If the $m$ significantly aggregated genes are effectively a random sample uniformly selected from set $\mathrm{B}$, the expected value of $m$ is $(n / N) M$. Because a gene can be selected only once, this is sampling without replacement and can therefore be appropriately modeled by a hypergeometric distribution [4]. The probability of observing at least $m$ significantly familially aggregated genes in the GO category of $n$ genes can be computed as follows:

$$
p=1-\sum_{i=0}^{m-1} \frac{\left(\begin{array}{l}
M \\
i
\end{array}\right)\left(\begin{array}{l}
N-M \\
n-i
\end{array}\right)}{\left(\begin{array}{l}
N \\
n
\end{array}\right)}
$$

The $p$-value calculated above corresponds to a one-sided test and a smaller $p$-value relates to a higher likelihood of a GO category's enrichment with genes that aggregate significantly in families. In this study, to avoid the possible loss of the true positives, we identified significant GO categories on the basis of the criterion of nominal significance of $p \leq 0.01$. Therefore, the $p$-value quoted should be considered as a heuristic measure, useful as an indicator that roughly rates the relative enrichment of significantly familially aggregated genes for each GO category. 


\section{Results \\ How do gene expressions aggregate among different types of relative pairs?}

Of 3300 genes evaluated, we found 1105 genes having one or more significant (empirical $p \leq 0.05$ ) familial correlation, and 212 genes having one or more highly significant $(p \leq 0.01)$ familial correlation. Table 1 shows the distributions of correlation estimates for the 3300 genes per the relative types. Sibling correlations were the highest, as expected by quantitative genetics theory, possibly due to a larger shared non-genetic component. Further examination of the pool of significant sibling correlations, revealed that about half of them (291 genes) were shared by the three types of sibling pairs, thus likely to be genderindependent. Also, the higher correlations between the more closely related pairs were in agreement with quantitative genetics theory [5]. It is interesting to observe that at the level of 0.01, the number of significant correlation estimates was dramatically reduced for all the relative types. We plotted the distributions of familial correlations for all 3300 genes per relative type. The distributions of correlations for the 15 types of relative pairs (Fig. 1) show that 1) the correlation estimates for brother-brother, sister-brother, and sister-sister were skewed to positive with few negative estimates; 2) much larger proportions of the estimates for the remaining 12 types were negative.

\section{How are familial correlations distributed (aggregated) over chromosomes?}

To answer the question, Figure 2 was made to visualize the aggregation of the genes with significant brother-brother correlations $(p \leq 0.05)$ on autosomes according to the relative physical distances of these genes. It can be easily seen that the genes are not uniformly distributed on the chromosomes and form at least one highly aggregated region on each chromosome. It is noteworthy to look at a highly aggregated 5-Mb band (29-34 Mb) on chromosome 6, which contains six genes, three of which have direct relevance to immunology. Genes HLA-DOA (Gene_ID 3111, 33082315-33085367) and HLA-F (Gene_ID 3134, 29801690-29802280) are important known immunological genes. TAP2 (GENE_ID 6891, 3290427532914499 ) is a neighbor of the HLA cluster, which encodes a protein participating in an antigen representation process. VARS (GENE_ID 7407, 3185327731871489 ) is an aminoacyl-tRNA synthetase. CLIC1 (GENE_ID 1192, 31806342-31812292) encodes chloride intracellular channel 1. A protein encoded by PPP1R10 (GENE_ID 5514, 30676161-30692987) has an inhibitory effect on protein phosphatase-1.

\section{How are significantly familially aggregated genes aggregating in function categories?}

We put all genes onto gene ontology (GO) [6] to get the categories. Then we selected the GO categories that contained at least five genes. Next, the hypergeometric test was applied to obtain a $p$-value of each studied category for its enrichment with significantly familially aggregated genes (i.e., from set B of 1105 genes). We found that more than one-third of the studied categories (36 out of 100 molecular function categories, 49 out of 119 biology process categories) were significantly enriched. However, taking into account the 229 categories evaluated, and using the very conservative Bonferroni correction, only six molecular function categories (GO categories one to six shown in Table 2) and four biological process categories (data not shown) remain significant. Table 2 lists the highly significantly (nominal $p \leq 0.01$ ) enriched GO molecular function categories. It is interesting to note that the majority of the enriched GO categories relate to

Table I: Numbers of significant familial correlations $(R)$

\begin{tabular}{|c|c|c|c|}
\hline \multirow[t]{2}{*}{ Type } & \multirow[t]{2}{*}{ Observed number of pairs } & \multirow{2}{*}{$\begin{array}{l}R>0.4609 \\
(P<0.05)\end{array}$} & \multirow{2}{*}{$\begin{array}{l}R>0.6532 \\
(P<0.01)\end{array}$} \\
\hline & & & \\
\hline Father-son & 57 & 36 & 0 \\
\hline Mother-son & 57 & 32 & 2 \\
\hline Father-daughter & 53 & 78 & 0 \\
\hline Mother-daughter & 53 & 20 & 1 \\
\hline Brother-brother & 105 & 532 & 48 \\
\hline Sister-brother & 179 & 676 & 104 \\
\hline Sister-sister & 94 & 688 & 161 \\
\hline Grandfather-father-grandson & 57 & 29 & 1 \\
\hline Grandmother-father-grandson & 57 & 19 & 0 \\
\hline Grandfather-mother-grandson & 57 & 34 & 0 \\
\hline Grandmother-mother-grandson & 57 & 37 & 3 \\
\hline Grandfather-father-granddaughter & 53 & 16 & I \\
\hline Grandmother-father-granddaughter & 53 & 19 & 0 \\
\hline Grandfather-mother-granddaughter & 53 & 48 & 2 \\
\hline Grandmother-mother-granddaughter & 53 & 69 & 3 \\
\hline
\end{tabular}




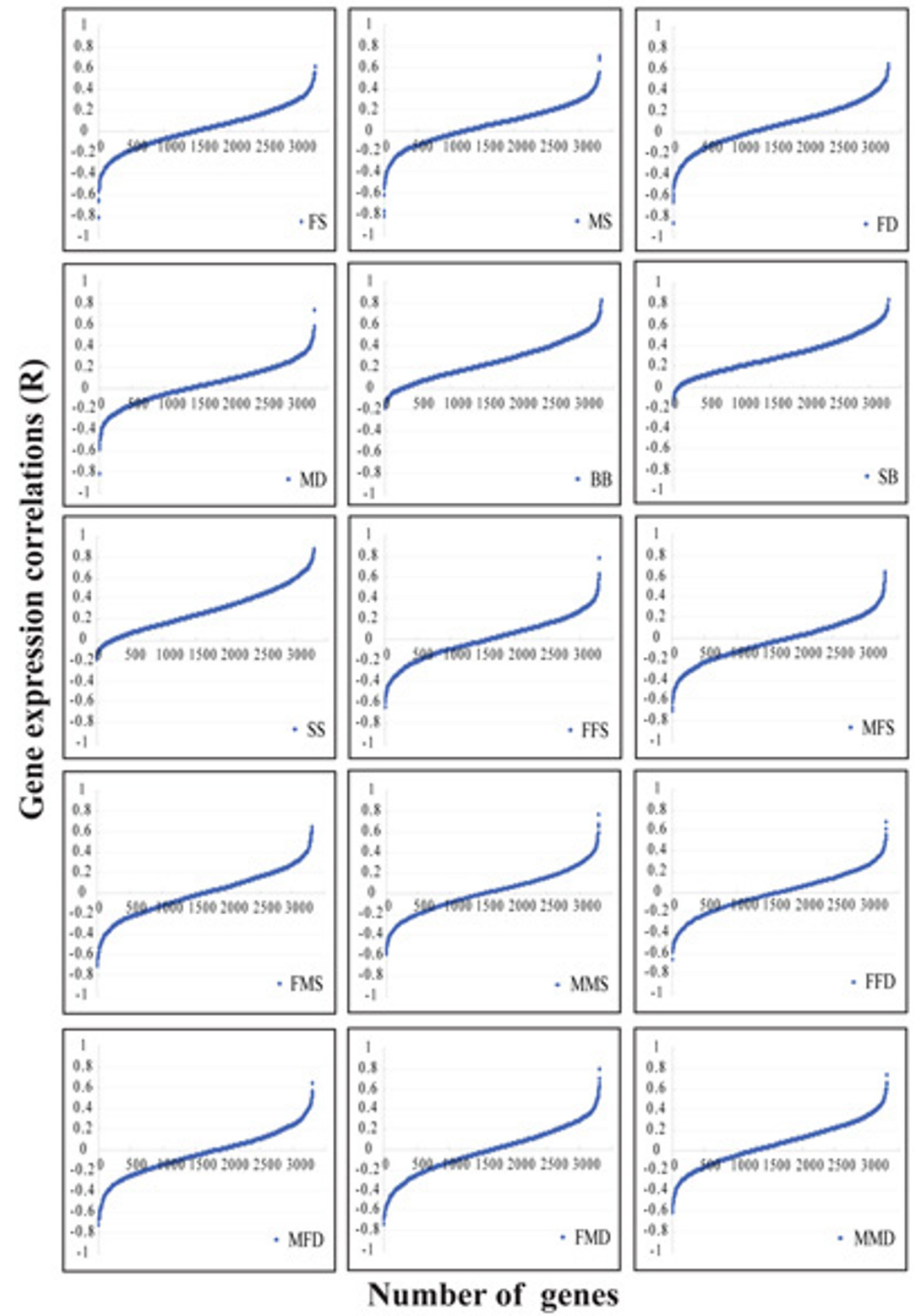

Figure I

Gene expression correlation distributions in different types of relative pairs. Each panel is for a type of relative pairs. The transverse axis stands for the ordered number of the 3300 studied genes and the longitudinal one denotes the expression correlations. 


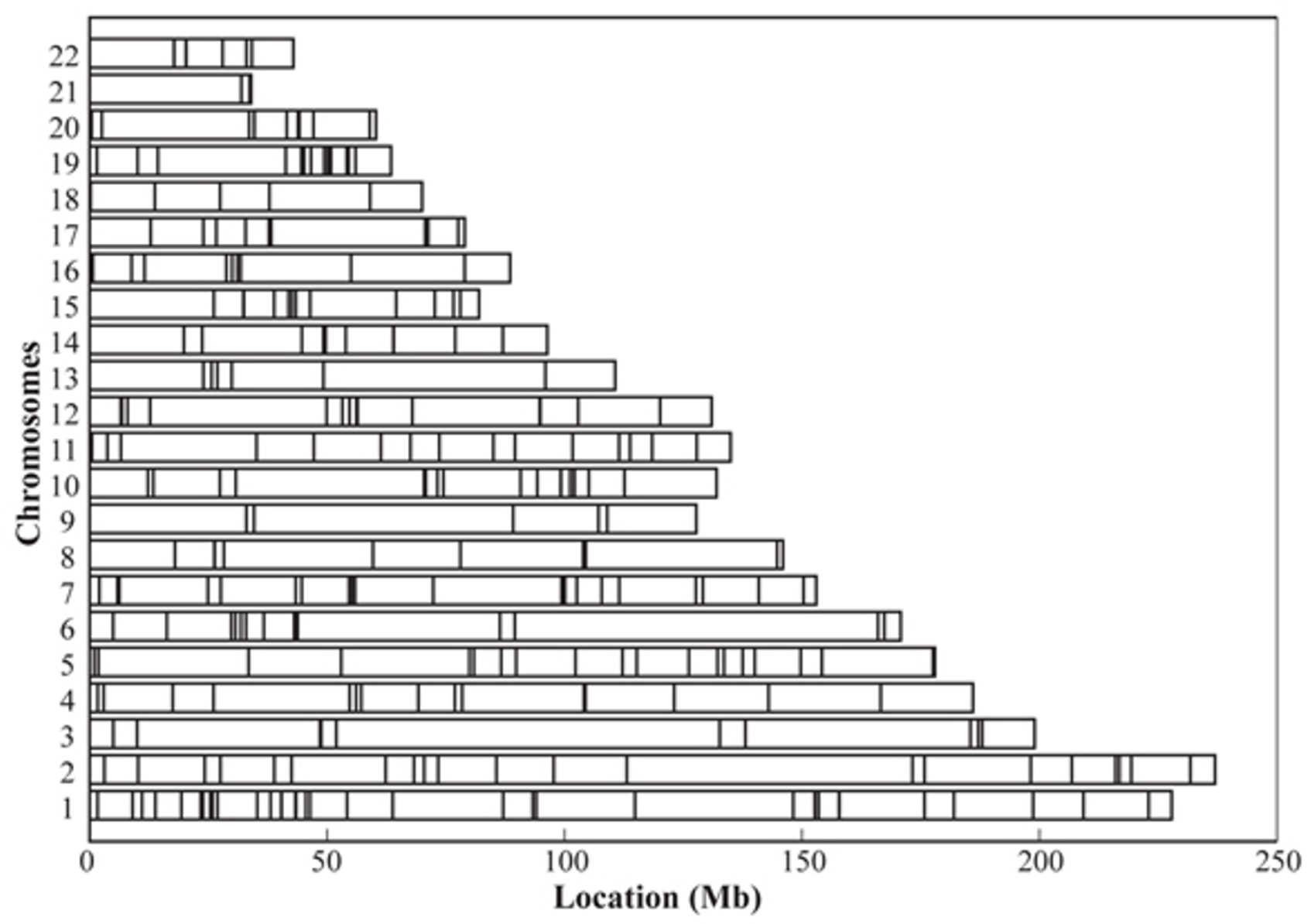

Figure 2

Distributions of familial (brother-brother) correlations over chromosomes. The horizontal bar is the relative length of chromosomes, and the vertical lines on chromosomes are used to indicate the approximate positions of the genes with significant BB correlations.

molecular binding, transcription factor, ligase activity, and receptor activity.

\section{Discussion}

To the best of our knowledge, this study is the first attempt to relate the familial aggregation patterns of genes with their genomic locations and their functionalities. Familial aggregation analysis of a large number of genes using different relative types suggests that some non-Mendelian genetic factors or environment factors may affect these gene expressions too, such as age-dependent genetic imprinting [7] or antagonistic environments for family members in different generations, possibly leading to biased estimates of some familial correlations. Regarding the use of different relative types for estimating additive genetic effects in gene expressions, it appears that no single relative type stands out as the best for all scenarios. The results acquired from this analysis of genome-wide gene expression traits raise a paradoxical challenge regarding the use of familial aggregation analysis to determine the genetic contribution to a quantitative trait. On one hand, the use of sibling pairs is favored because it is unlikely to produce a negative estimate of heritability, but tends to overestimate it because of the larger shared non-genetic components and dominance components. On the other hand, the use of other relative pairs is unlikely to overestimate heritability, but can be problematic if some factor(s) (e.g., antagonistic environments) causes the familial individuals between different generations to be environmentally negatively correlated.

Further bioinformatics analysis of familial aggregated genes suggests some consistencies between familial aggregations and chromosomal aggregations and functional aggregations. However, we feel that these exploratory results warrant further investigation because of the limited 
Table 2: A list of GO molecular function categories highly significantly $(p \leq 0.01)$ enriched with genes of high familial aggregations

\begin{tabular}{lllll}
\hline GO ID & GO Description & $n^{\mathrm{a}}$ & $m^{\mathrm{b}}$ & $p$ \\
\hline GO:0000166 & nucleotide binding & 468 & 202 & $6.15 \times 10^{-7}$ \\
GO:0003735 & structural constituent of ribosome & 54 & 5 & $2.5 \times 10^{-5}$ \\
GO:0003723 & RNA binding & 177 & 83 & $4.64 \times 10^{-5}$ \\
GO:0004872 & receptor activity & 174 & 37 & $9.95 \times 10^{-5}$ \\
GO:0005509 & calcium ion binding & 150 & 31 & 0.000154 \\
GO:0051082 & unfolded protein binding & 66 & 36 & 0.000187 \\
GO:0005524 & ATP binding & 377 & 152 & 0.000578 \\
GO:0005515 & protein binding & 880 & 264 & 0.001239 \\
GO:0004004 & ATP-dependent RNA helicase activity & 6 & 6 & 0.001397 \\
GO:0016874 & ligase activity & 73 & 35 & 0.003392 \\
GO:0003779 & actin binding & 57 & 10 & 0.003461 \\
GO:0003700 & transcription factor activity & 232 & 63 & 0.005974 \\
GO:0004842 & ubiquitin-protein ligase activity & 31 & 17 & 0.007186 \\
GO:0003676 & nucleic acid binding & 80 & 36 & 0.008773 \\
GO:0005506 & iron ion binding & 57 & 27 & 0.009803
\end{tabular}

an, Number of genes contained in a category identified and counted by using set $A$ (a total of 3300 genes).

${ }^{b} m$, Number of (highly familially aggregated) genes contained in the category, counted using set B (a total of II 05 genes).

sample size used in the study. In addition, traditional quantitative genetics approaches, which assume a polygenic basis for the studied traits and normal distribution of the underlying genetic effects, might not be the most appropriate to analyze the expression phenotypes whose genetic models could be monogenic or oligogenic. Although the familial aggregation analysis approach as implemented in S.A.G.E. is robust to non-normality of traits, further study is needed regarding our method's behaviors and properties when applied to traits having a genetic basis quite deviated from what is expected for truly quantitative traits.

\section{Conclusion}

Most of our results from the genetic epidemiological analysis were consistent with quantitative genetics theory. Further bioinformatics analysis revealed that familially aggregated genes tended to aggregate on some genomic regions and to enrich their functional categories.

\section{Competing interests}

The author(s) declare that they have no competing interests.

\section{Acknowledgements}

This work was supported in part by the National Natural Science Foundation of China (Grants 301705I5, 30370798, 3057I034, 30570424, 30600367, and 30670484) and U.S. National Institutes of Health SCCOR grant (P50 HL077 IOI-OI). Some of the results were obtained using the program package S.A.G.E., which is supported by a U.S. Public Health Service Resource Grant (RR03655) from the National Center for Research Resources.

This article has been published as part of BMC Proceedings Volume I Supplement I, 2007: Genetic Analysis Workshop 15: Gene Expression Analysis and Approaches to Detecting Multiple Functional Loci. The full contents of the supplement are available online at http://www.biomedcentral.com/ $|753-656| / \mid$ ? issue=SI.

\section{References}

I. Khoury MJ, Beaty TH, Kung-Yee L: Can familial aggregation of disease be explained by familial aggregation of environmental risk factors? Am J Epidemiol 1988, I 27:674-683.

2. Morley M, Molony CM, Weber TM, Devlin JL, Ewens KG, Spielman RS, Cheung VG: Genetic analysis of genome-wide variation in human gene expression. Nature 2004, 430:743-747.

3. Statistical Analysis for Genetic Epidemiology, Release 5.3 [http://genepi.cwru.edu/]

4. Guo Z, Zhang T, Li X, Wang Q, Xu J, Yu H, Zhu J, Wang H, Wang C, Topol EJ, Wang $Q$, Rao S: Towards precise classification of cancers based on robust gene functional expression profiles. BMC Bioinformatics 2005, 6:58.

5. Falconer DS, Mackay TFC: Introduction to Quantitative Genetics 4th edition. London: Longman; 1996.

6. Ashburner M, Ball CA, Blake JA, Botstein D, Butler H, Cherry JM, Davis AP, Dolinski K, Dwight SS, Eppig JT, Harris MA, Hill DP, IsselTarver L, Kasarskis A, Lewis S, Matese JC, Richardson JE, Ringwald M, Rubin GM, Sherlock G: Gene ontology: tool for the unification of biology. The Gene Ontology Consortium. Nat Genet 2000, 25:25-29.

7. Bjornsson HT, Fallin MD, Feinberg AP: An integrated epigenetic and genetic approach to common human disease. Trends Genet 2004, 20:350-358.

Publish with Biomed Central and every scientist can read your work free of charge

"BioMed Central will be the most significant development for disseminating the results of biomedical research in our lifetime. "

Sir Paul Nurse, Cancer Research UK

Your research papers will be:

- available free of charge to the entire biomedical community

- peer reviewed and published immediately upon acceptance

- cited in PubMed and archived on PubMed Central

- yours - you keep the copyright 